\title{
Attitudes of paediatricians to HIV and hepatitis B virus infection
}

\author{
P W Buss, M McCabe, E R Verrier Jones
}

\begin{abstract}
There is a recent upsurge of interest among health care professionals regarding the risk of accidental occupational exposure to HIV virus. We evaluated knowledge of virus carriage prevalence, needlestick injuries, venepuncture practices, and glove use among paediatricians in Wales and South West Regional Health Authorities. We also attempted to evaluate hepatitis $B$ immunisation uptake in this group. Paediatricians have traditionally been considered a low risk group in the context of accidental occupational exposure to these viruses.
\end{abstract}

We targeted a four point questionnaire at 221 paediatricians. Results suggested that despite recent increasing concern about these viruses, that is reflected in the amount of medical literature recently published, and the issuing of Department of Health guidelines on venepuncture, knowledge of prevalence of HIV and hepatitis B carriage rates, and hence assessment of risk magnitude, was surprisingly poor. Safe venepuncture practices were not widely used. In the 12 months before receiving the questionnaire $55 \%$ had suffered a needlestick injury with only $10 \%$ reporting the fact. Hepatitis B immunisation uptake was highest in the junior grades (but this does not necessarily mean those at greatest risk). There were many inconsistencies between the clinicians' perceptions of risk and their practices.

As the virus attains a firm hold in the heterosexual population paediatricians by virtue of the nature of venepuncture in children will almost certainly see their risk of acquiring HIV/hepatitis B viruses secondary to accidental occupational exposure increase over the next decade. Without an improvement in current knowledge of carriage prevalence in high risk areas and alteration in venepuncture practices/hepatitis B immunisation uptake some will unfortunately, though avoidably, contract these bloodborne viral infections.

Accidental occupational exposure to bloodborne viruses including $\mathrm{HIV} /$ hepatitis $B$ is to be increasingly expected as investigative medicine proceeds and more difficult procedures are undertaken. There has been a recent upsurge in interest of the hazards of accidental occupational exposure to these viruses among surgeons $^{12}$ and anaesthetists. ${ }^{3}$

These two groups have traditionally been considered the groups most at risk of acquiring these viruses secondary to accidental occupational exposure. Paediatricians have been considered to be among those at low risk with very little work having actually been undertaken in this group.

There are improved venepuncture techniques now more widely used among adults and difficulties in using gloves in paediatric practice. Because of this and because of recent evidence that HIV is occurring increasingly among the heterosexual population, ${ }^{4}$ it was felt that paediatricians may well find themselves at increased relative risk.

A survey was undertaken by questionnaire of paediatricians from hospitals accepting acute paediatric admissions in Wales and South West Regional Health Authorities. The aim of the questionnaire was to assess the following four aspects. (1) Knowledge of prevalence of HIV seropositivity in a high risk area and hepatitis carriage rate (an assessment of risk magnitude). (2) Needlestick injury: frequency of and related actions of the paediatrician. (3) Venepuncture practice: including the use of gloves in clinical practices. (4) Hepatitis B immunisation uptake among paediatricians.

\section{Method}

The questionnaire was targeted at 221 paediatricians in two regions, the Wales and South West Regional Health Authorities. A single mailing was undertaken without reminders. The questionnaire was designed so that answering entailed encircling or underlining the answer the paediatricians felt to be correct.

\section{QUESTIONNAIRE (SEE APPENDIX)}

A total of 20 questions (17 stem questions) were posed and this included two questions asking about perception of current risk and whether or not this was likely to increase. This enabled us to divide the assessment of results into two groups (in fact of similar size and with a similar ratio of staff from all grades).

\section{Results}

We received a reply from $127(57 \cdot 5 \%)$ of the 221 paediatricians in the two regions. There were 31 consultants $(24.5 \%), 27$ paediatricians of middle grade $(21 \cdot 0 \%)$, and 69 senior house officers or junior grade $(54.5 \%)$. This return rate is quite consistent with previous mail surveys reported by Cartwright among hospital medical personnel. ${ }^{5}$ Our survey was based on a single mail shot with no reminders. The decision not to send reminders was based on two reasons. (1) 
Internal rotation of staff may well have taken place and as the survey was anonymous then there was a real risk of data duplication. (2) A short term perception of disease risk was necessary to examine practices in those groups with different risk perceptions. With an aggressive public education campaign occurring throughout the latter part of the last year, long term risk perception may well have been altered and hence affected analysis of results.

The knowledge of HIV seropositivity prevalence in high risk areas was very poor with only $23 \%$ correct overall. The highest number of correct answers was among middle grades $(30 \%)$ and lowest among senior house officers (17\%). A similar result was obtained for prevalence of hepatitis B carriage. Of the total questioned $93 \%$ confirmed the greater infectivity of hepatitis B compared with HIV (97\% among senior hours officers compared with $87 \%$ among consultant grade staff) (see table 1).

Needlestick injuries had been suffered by $85 \%$ of those questioned sometime during their career and $55 \%$ in the last year. Many had suffered more than one needlestick injury but only seven out of $70(10 \%)$ reported these events. Of those that reported these events only four out of seven $(57 \%)$ had had antibody concentrations checked. Needlestick injuries were equally common among junior and middle grade staff.

General glove usage was low in general paediatric settings (35\% of total sometimes/ always wore gloves). They were used most in the higher age group children. Gloves were frequently used in accident and emergency departments but used most in the delivery suite area (56 out of $114(49 \%)$ in accident and emergency and 91 out of $112(81 \%)$ in the delivery suite).
This suggests that safety measures take up a much higher profile in these settings. When asked about risk perception at the present time 55 out of $127(43 \%)$ felt it was low and increasing whereas 48 out of $127(38 \%)$ felt it was high and increasing (see tables 2 and 3 ).

Unfortunately resheathing of needles was always/sometimes undertaken by 101 out of 126 (80\%): 40/126 (32\%) always and 61/126 (48\%) sometimes. This put the doctors at very high risk of accidental occupational exposure. Formal instruction on phlebotomy technique had been received by 38 out of $127(30 \%)$. In the high risk perception group, interestingly, only $17 \%$ had received formal instruction (compared with $40 \%$ in those who perceive a low current risk).

Overall hepatitis B immunisation uptake was 90 out of $127(71 \%)$ of paediatricians. However, this represents an immunisation uptake of $88 \%$ among senior house officers compared with $60 \%$ of middle grade staff and $42 \%$ consultants. Only 58 out of $90(64 \%)$ of those immunised had had antibody concentrations checked. The reasons for non-immunisation were varied and were not clear.

Nearly all (95\%) paediatricians expected their relative risk of acquiring $\mathrm{HIV} /$ hepatitis $B$ as opposed to other groups to increase. This was against a group of $38 \%$ who already perceived themselves to be at high risk of acquiring hepatitis B secondary to accidental occupational exposure.

When looking at the group who already perceived a high risk (and which would increase in the future) the following was found. (1) Altogether $56 \%$ had had needlestick injury in the last year but only $11 \%$ of these had reported it (compared with $51 \%$ and $14 \%$ reporting in low

Table 1 Paediatricians' assessment of risk magnitude and knowledge of HIV seropositivity in high risk area and hepatitis $B$ carriage in the UK. Results are number giving correct answer/number of respondents (\%)

\begin{tabular}{llllr}
\hline & Consultant & Middle grade & Senior house officer & Total \\
\hline Prevalence of hepatitis B carriage & $10 / 31(32)$ & $12 / 27(44)$ & $19 / 69(28)$ & $41 / 127(32)$ \\
Prevalence of HIV seropositivity & $9 / 31(29)$ & $8 / 27(30)$ & $12 / 69(17)$ & $29 / 127(23)$ \\
Infectivity of HIV/hepatitis B & $27 / 31(87)$ & $23 / 26(88)$ & $66 / 68(97)$ & $116 / 125(93)$ \\
\hline
\end{tabular}

Table 2 Evaluation of needlestick injury and safety practices amongst paediatricians. ${ }^{*}$ Results for glove usage are number who sometimes or always wore gloves/number of respondents (\%)

\begin{tabular}{|c|c|c|c|c|c|c|c|}
\hline \multirow[t]{2}{*}{ Risk perception } & \multirow{2}{*}{$\begin{array}{l}\text { No (\%) who } \\
\text { had needlestick } \\
\text { injury } \\
\text { (in one year) }\end{array}$} & \multirow{2}{*}{$\begin{array}{l}\text { No (\%) of those } \\
\text { with needlestick } \\
\text { injury who } \\
\text { reported it }\end{array}$} & \multicolumn{5}{|c|}{ Glove usage at different ages* } \\
\hline & & & Delivery & Neonatal & Infant & Child & $\begin{array}{l}\text { Accident and } \\
\text { emergency }\end{array}$ \\
\hline $\begin{array}{l}\text { High (current) and } \\
\text { increasing }(n=48)\end{array}$ & $27(56)$ & $3(11)$ & $35 / 43(81)$ & $14 / 47(30)$ & $17 / 48(35)$ & $21 / 48(43)$ & $24 / 42(57)$ \\
\hline $\begin{array}{l}\text { Low (current) and } \\
\text { increasing }(n=55)\end{array}$ & $28(51)$ & $4(14)$ & $40 / 49(81)$ & $19 / 52(36)$ & $20 / 52(38)$ & $22 / 53(41)$ & $21 / 52(40)$ \\
\hline
\end{tabular}

Table 3 Evaluation of needlestick injury and safety practices among paediatricians

\begin{tabular}{|c|c|c|c|c|}
\hline Risk perception & $\begin{array}{l}\text { No (\%) who used } \\
\text { Vacutainers* }\end{array}$ & $\begin{array}{l}\text { No (\%) who had } \\
\text { phlebotomy instruction }\end{array}$ & $\begin{array}{l}\text { No }(\%) \text { who } \\
\text { resheathed needles }\end{array}$ & $\begin{array}{l}\text { No (\%) who had } \\
\text { hepatitis B } \\
\text { immunisation }\end{array}$ \\
\hline High (current) and increasing $(n=48)$ & $13(27)$ & $8(17)$ & $\left.\begin{array}{l}12 \text { (always) } \\
22 \text { (sometimes) }\end{array}\right\}(71)$ & $37(77)$ \\
\hline Low (current) and increasing $(n=55)$ & $18(33) \dagger$ & $22(40)$ & $\left.\begin{array}{l}19 \text { (always) } \\
26 \text { (sometimes) }\end{array}\right\}(82)$ & $40(73)$ \\
\hline
\end{tabular}

*Becton-Dickinson.

tOnly 54 respondents. 
risk group); $\chi^{2}=0.294, p>0.05$. (2) A total of $77 \%$ had received hepatitis $B$ immunisation (compared with $73 \%$ in those who perceived a low risk); $\chi^{2}=0 \cdot 26, p>0 \cdot 05$. (3) Altogether $39 \%$ always/sometimes used gloves in general paediatric settings (compared with $41 \%$ in low risk group); $\chi^{2}=0.0087, p>0.05$. (4) A total of $71 \%$ resheathed needles (compared with $81 \%$ in low risk group); $\chi^{2}=1 \cdot 73, p>0.05$. Statistical analysis of these figures showed no difference between the groups.

\section{Discussion}

As clinicians involved in paediatric practice we, the authors, felt that an assessment of this nature was long overdue. With changes in adult venepuncture practices occurring during the last decade, though not easily applicable to paediatric venepuncture, it was felt that paediatricians largely seem to ignore the risks that venepuncture poses to themselves.

Very little work has been done among paediatricians, though they were mentioned in work by Levy et al. ${ }^{6}$ Generally they have been considered to be' a low risk group with much lower risks than surgeons, anaesthetists, and nursing staff in accident and emergency departments. This may well remain the case at present, though with changing epidemiological characteristics of HIV and more sophisticated techniques used among small infants and children in intensive care settings the risks may well alter. The current world hepatitis B carriage rate is in the order of 200 million people ${ }^{7}$ with the UK carriage rate in the order of $0.1-0.5 \%{ }^{8}$ Seroconversion after contaminated needlestick injury with hepatitis $B$ is in the range of $5 \%{ }^{9}$ and $30 \%{ }^{10}$ and for HIV in the order of $0.5 \%$. ${ }^{11}$ This must be considered against a background of increasing numbers of invasive procedures undertaken in children and the all too often sight of the junior paediatrician learning the trade with a hand covered in blood from his first few heelprick attempts. In many ways this scenario (prolonged and frequent skin contact with blood) is as disturbing as the failure to report needlestick injury. ${ }^{6}$

The lack of uniformity of HIV prevalence meant it was difficult to choose a representative figure. We therefore (in view of the fact that we were assessing paediatricians) chose the survey of Guthrie test analysis by Peckham et al in a high risk area. ${ }^{12}$ This put a seroprevalence figure of $0 \cdot 24 / 1000$ which represented the at risk population to a paediatrician practising in this region in the delivery suite but not the figure for true HIV infection. ${ }^{13}$ This was also not the figure that any of the paediatricians we assessed were facing but with a continuous change of staff (particularly junior grade and locum staff) a junior doctor could find himself working in a high risk setting at any time in his career.

There has been quite widespread education in the media and by occupational physicians both nationally and in the relevant areas over the last few years. Our results, however, suggest that knowledge pertaining to HIV seropositivity and hepatitis B carriage (and hence magnitude of risk assessment) is poor. More than two thirds of our respondents were out by a factor of 10 or more in their estimation of prevalence. This contrasts quite considerably with the better general knowledge that health care workers have compared with the public regarding HIV transmissibility, etc. ${ }^{14}$ Disturbingly venepuncture practice among all groups of paediatricians leaves a lot to be desired.

Despite recent issuing of guidelines from the Department of Health regarding venepuncture ${ }^{9}$ more than $75 \%$ of junior paediatricians still resheath needles. This if reflected nationally would put many at risk of HIV in the future as well as hepatitis B (as even in those immunised there is a $4 \%-10 \%$ risk of attainment of inadequate antibody concentrations). ${ }^{15} 16$

The universal failure to report needlestick injury must surely be improved with strenuous attempts by occupational physicians to get the message across. We are aware of a similar situation in the USA where McCormick and Maki noted the apparent failure of physicians to report needlestick injury in 1981. Over a four year period in a large American centre with a turnover of 500 house grade staff annually only one needlestick injury was reported. ${ }^{17}$ Adequate time must be allowed from routine work to attend the occupational physician and obtain advice after a needlestick injury. The actual reasons for both non-reporting needlestick injury and non-hepatitis B immunisation uptake are not clear. In our study it appeared that higher risk perception did not necessarily mean safer venepuncture practices.

Protective measures such as hepatitis B immunisation is low among those most often asked to undertake the difficult procedure. This needs to be corrected and a lesson learnt from the more junior counterparts. Hepatitis B immunisation alone is not sufficient reason for not reporting needlestick injury (it should not engender a feeling of false security).

Usage of gloves must be encouraged (they can protect against blood contact even if they have limited use in preventing needlestick injury) in all potential high risk situations and in all difficult procedures. The design of a thin but durable glove for the use of neonatal paediatricians would be helpful. The problem of universal implementation is likely to be one of cost (possibly an overall $40 \%$ increase in isolation material budget for universal implementation). ${ }^{18}$ It is most desirable that juniors get simple clear instruction on venepuncture technique from their senior colleagues and an experienced nurse helps to hold the child especially in the first few weeks of a job.

It has been estimated that approximately $2 \%$ of people infected with hepatitis B die of the infection, ${ }^{19}$ and one half of those infected with HIV will have developed AIDS within six years. ${ }^{20}$ Hopefully this is enough of a risk for paediatricians to take note and alter their venepuncture practices, hepatitis B immunisation status, and basic attitudes to these viruses in the workplace. The danger is that paediatricians will still consider hepatitis B and HIV as 'adult diseases' for much too long before altering prac- 
tices that will put themselves at risk of acquiring these viruses secondary to accidental occupational exposure. We hope that this limited study will have highlighted the problem facing paediatricians as regards to their attitudes to these viruses in the workplace and will lead the way for further more extensive studies of the subject.

1 Gazzard BG. HIV and surgeons. BMF 1990;301:1003-4

$2 \mathrm{LeF}$ Porteus MJ. Operating practices of the precaution taken by orthopaedic surgeons to avoid infection with HIV taken by orthopaedic surgeons to avoid
and hepatitis B. BMf 1990;301:167-9.

3 Editorial. Occupational infection amongst anaesthetists. Lancet 1990;336:1103.

4 Norman S, Sludd J. HIV infection and women. BMF 1990; 301:1231-2.

5 Cartwright $A$. Professionals as poor responders; variations in an effects of response rates to questionnaires 1961-77. BMJ 1978;ii:1419-21.

6 Levy B, Harris JC, Smith JL, et al. Hepatitis B in ward and clinical laboratory workers in a general hospital. $A m$ f Epidemiol 1977;106:330-5.

7 Zuckermann A. Who should be immunised against hepatitis B. $B M \mathcal{F} 1984 ; 289: 1243$.

8 Association of Anaesthetists. AIDS and hepatitis B-guide- lines for anaesthetists. London: Association of Anaesthetists, April 1988:5.

9 Recommendations of the Expert Advisory Group on AIDS. Guidance for clinical health care workers-protection against infection with HIV/hepatitis B. London: HMSO, 1990.

10 Centers for Disease Control guidelines for prevention of HIV and hepatitis B viruses to health care and public safety workers. MMWR 1989;38(suppl 56, 4-5)31-3.

11 Ruthane $M$. Surveillance of health care workers exposed to blood from patients exposed to HIV virus. $N$ Engl $\mathcal{F}$ Med 1988;319:1118-23.

12 Peckham CS, Tedder RS, Briggs M, et al. Prevalence of maternal HIV infection based on unlinked anonymous testing in newborn babies. Lancet 1990;335:516-9.

13 European Collaborative Study. Mother to child transmission of HIV infection. Lancet 1988;ii:1039-43.

14 Smithien R. Public health staff knowledge about AIDS. Community Med 1988;10:221-7.

15 Eddleston LA. Modern vaccines-hepatitis. Lancet 1990;335. 1142.

16 Scully C. Uptake of hepatitis B immunisation amongst UK dental undergraduates. Health Trends 1990;22:92.

7 McCormick RD, Maki DG. Epidemiology of needlestick injuries in hospital personnel. Am f Med 1981;70:928-32.

18 Doebbling BN, Wenzel RP. The direct cost of universal precautions in a teaching hospital. JAMA 1990;264: 2083-7.

9 Morgan DR. HIV and needlestick injuries. Lancet 1990;335: 1280

20 Moss AR, Baccheti P, Osmond D, et al. Seropositivity for HIV and the development of AIDS or AIDS related condition: three year follow up of San Francisco General Hospital cohort. BMF 1988;296:745-50.

Appendix

Study of venepuncture technique, knowledge of factors affecting occupational exposure to hepatitis B and HIV virus, and uptake of hepatitis $B$ vaccination among paediatricians in Wales and South West England

Current position in NHS:

Please underline or encircle the answer(s) you feel to be correct

Hepatitis $\mathrm{B} / \mathrm{HIV}$ virus:

(1) The current estimate of hepatitis B carrier in the UK at present is in the order of:

(1) 1 in 1000

(2) 1 in 10000

(3) 1 in 100000

(1) 1 in 5000

(2) 1 in 50000

(3) 1 in 500000 seropositivity in 114515 Guthrie tests showed a positive rate in the Thames' regions of approx:

(3) Which virus is thought to have higher infectivity? HIV/hepatitis B

(4) As a paediatrician my current risk of attaining hepatitis B secondary to accidental occupational exposure is (relative to other hospital doctors):

(1) Low

(2) High

(3) Don't know

Needlestick injuries:

(1) Have you ever had a needlestick injury?

(2) How many have you received in the last year?

(3) Did you report these incidents?

(4) If so, was action taken?

Yes/no

1/2/over 3

Yes/no

(1) No

(2) Yes, hepatitis B antibody

(3) Yes, hepatitis B immunisation

(4) Yes, HIV antibody checked

Phlebotomy technique:

(1) When taking blood do you use gloves:

(a) In neonates

(b) In infants

(c) In children

(2) Have you received instructions in phlebotomy technique?

(3) When taking blood do you use the Vacutainer system in infants/children?

(4) When attending a delivery on delivery suite, do you wear gloves?

(5) When attending an injured child in the accident department, do you wear gloves?

(6) Do you resheath needles after use?

Hepatitis B immunisation:

(1) Have you received hepatitis B immunisation?

(2) If no to (1), is the reason for this?

(3) If yes to (1) have antibody levels been checked?

Summary:

Do you feel that the relative risk to paediatricians of hepatitis B/HIV, as opposed to other groups, will alter in the next decade?

Always/sometimes/never Always/sometimes/never Always/sometimes/never Yes/no

Always/sometimes/never

Always/sometimes/never

Always/sometimes/never

Always/sometimes/never

Yes/no

(1) 'Can't be bothered'

(2) See little risk

(3) Worried regarding vaccine

(4) Never offered vaccine

(5) Other

Yes/no

(1) Yes, it will increase

(2) Yes, it will decrease

(3) $\mathrm{No}$ 